\title{
Enhancement of graft survival and sensorimotor behavioral recovery in rats undergoing transplantation with dopaminergic cells exposed to glial cell line-derived neurotrophic factor
}

\author{
Vivek Mehta, M.D., Murray Hong, Ph.D., Julian Spears, B.Sc., and Ivar Mendez, M.D., Ph.D. \\ Neural Transplantation Laboratory, Department of Surgery, Division of Neurosurgery, Dalhousie \\ University, Halifax, Nova Scotia, Canada
}

The goal of this study was to investigate the ability of fetal dopaminergic neurons to improve complex sensorimotor behavior.

The authors obtained ventral mesencephalic tissue from 14-day-old rat fetuses. The cells were exposed to glial cell line-derived neurotrophic factor (GDNF) prior to transplantation into rats with unilateral 6-hydroxydopamine lesions of the dopaminergic nigrostriatal pathway. Animals that received 400,000 cells exposed to GDNF demonstrated significant improvement in contralateral forelimb function and showed improvement in rotational behavior faster than animals that received cells not exposed to GDNF. Increasing the number of implanted cells to 800,000 exposed to GDNF did not result in any additional improvement in functional recovery.

As neural grafting procedures in the nervous system evolve and genetically engineered cells or stem cells replace fetal tissue, crucial questions about cell number and trophic regulation will need to be addressed. This study demonstrates that grafting of 400,000 cells exposed to GDNF before transplantation has a beneficial effect in the restoration of complex sensorimotor behavior.

Key Words * Parkinson's disease * neural transplantation * glial cell line-derived neurotrophic factor * graft survival * rat

Parkinson's disease (PD) is a progressive neurodegenerative disorder for which currently there are no treatment options that are curative. Fetal transplantation is a process whereby dopaminergic tissue is placed into the striatum of patients with PD in an attempt to replenish striatal dopamine and reconstitute, at least in part, the normal nigrostriatal circuitry. The success of fetal transplantation in both rodent and primate parkinsonian models has led to its experimental clinical application.[6,26-28] Although the early results of these clinical trials are promising, clinical improvement has not reached the level necessary for fetal transplantation to be used routinely as a therapeutic procedure in patients with PD.[5,7,31] Crucial shortcomings of grafting fetal cells center around poor cell survival, limited host reinnervation, and the inability of grafts to improve complex sensorimotor deficits in patients who have PD. Although the 
underlying reason for the poor survival rates of transplanted human dopaminergic neurons is not known, it is likely that cell loss occurs during storage, cell preparation, and the implantation procedure of dopaminergic cells.

In the rodent parkinsonian model, grafting of fetal dopaminergic cells improves rotational behavior bias; however, the extent of graft-derived benefit for more complex sensorimotor deficits such as forelimb akinesia has been limited.[18,24,26] Demonstration of improvement in these tests is relevant because the purpose of grafting is to ameliorate complex motor dysfunction in patients with PD. Recent evidence supporting the ability of growth factors to protect and enhance neuronal outgrowth has led to the possibility of using such factors in conjunction with neural transplantation for the treatment of degenerative disorders such as PD. The newest member of the transforming factor-beta superfamily, glial cell line-derived neurotrophic factor (GDNF), is a potent neurotrophin that has demonstrated the ability to increase midbrain dopamine levels, protect dopaminergic neurons, and increase fiber outgrowth both in vitro and in vivo. $[8,11,12,14,15,25]$

We have recently demonstrated that storage of fetal dopaminergic cells in a GDNF-rich hibernation medium for 6 days enhances graft survival.[2] We now postulate that "priming" these dopaminergic cells before transplantation in a GDNF-rich medium may also enhance complex sensorimotor functional recovery in the rodent parkinsonian model. To test this hypothesis we set out to determine the number of cells hibernated with or without GDNF that may be required to improve both rotational bias as well as integrated forelimb function in the rodent parkinsonian model.[18,24]

\section{MATERIALS AND METHODS}

\section{Animal Preparation}

Twenty-five adult female albino Wistar rats weighing 200 to $225 \mathrm{~g}$ each were used. The animals were housed two per cage with access to food and water ad libitum and allowed to acclimatize for 7 days in the animal care facility before being trained. The animals were maintained in a temperature-controlled environment in a 12-hour light/dark cycle. All animal procedures were performed in accordance with guidelines of the Canadian Council on Animal Care and the University Council on Laboratory Animals.

\section{Experimental Design}

The animals were divided into four groups. Group 1 consisted of seven rats that received grafts of 400,000 ventral mesencephalic cells. Group 2 was composed of seven rats that received grafts of 400,000 cells exposed to GDNF. The seven Group 3 rats received grafts of 800,000 cells exposed to GDNF. Group 4 was the control group and consisted of four animals that were lesioned by administration of 6-hydroxydopamine (6-OHDA), but did not receive a graft.

\section{Nigral Lesions}

The rats received two stereotactic injections of 6-OHDA (3.6 $\mu \mathrm{g}$ 6-OHDA hydrobromide/ $\mu \mathrm{l}$ in $0.2 \mathrm{mg} / \mathrm{ml}$ L-ascorbate) into the right nigrostriatal dopaminergic pathway after anesthesia had been induced by a ketamine-xylazine-acepromazine mixture. The injections were administered by using a 10- $\mu$ l Hamilton syringe at the following two coordinates: 1) anteroposterior (AP) -4.4, lateral (L) 1.2, ventral (V) 7.8, and toothbar -2.4; and 2) AP -4, L 0.8, V 8, and toothbar + 3.4. At the first coordinate site, $2.5 \mu$ of 6-OHDA

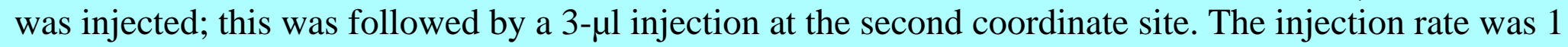
$\mu \mathrm{l} /$ minute and the cannula was left in place for 5 minutes before it was removed. After a 2-week recovery 
period, the animals were given an amphetamine challenge ( $5 \mathrm{mg} / \mathrm{kg}$ administered intraperitoneally) and only animals that exhibited 480 or more ipsilateral rotations in a 60 -minute period (> 8 rotations/minute) were included in the study.

\section{Behavioral Testing}

Rotational Behavior. Following transplantation, the animals were tested for rotational behavior using the computerized video activity monitoring system that was programmed for rotational behavior. Rotation behavioral testing was performed in 60-minute periods every 3 weeks after transplantation.

Sensorimotor Testing. All of the animals were first trained in the "staircase test" and "stepping test" (initiation, ramp time, and adjusting step) for a period of 2 weeks before being subjected to unilateral 6-OHDA lesioning. All behavioral tests were conduced by the same investigator in a consistent manner with regard to technique and time of testing. The animals were tested 2 weeks after unilateral 6-OHDA nigral lesions had been made and 8 weeks following graft transplantation. Each animal had four sessions in the staircase test and eight trials in all facets of the stepping test.

The staircase test was used to assess skilled forelimb use in the rat. All animals were deprived of food for a period of 24 hours and then placed in the staircase apparatus.[18] Briefly, the staircase apparatus consists of a plastic box that has two staircases with five steps each. The staircases are separated from each other in such a way that it is impossible for the animal to reach the staircase on the right with any limb other than the right forelimb or to reach the staircase on the left with any limb other than the left forelimb. The steps on each staircase were loaded with small food pellets, one pellet per step. The animals were allowed to attempt to acquire as many food pellets as possible using each forelimb independently within a 15-minute period. The number of pellets consumed by the rats was counted after each test period. Scores were assigned after both 1-minute and 15-minute intervals for both the left and right forelimb.

For the stepping test, a wooden ramp, $0.9 \mathrm{~m}$ long, was constructed and attached to the side of the rodent's home cage. The stepping test[24] consisted of three components: initiation time, ramp time, and adjusting step. The rats were immobilized in such a way that both hindlimbs and one forelimb were secured and the initiation to run up the ramp was done by either the right or the left forelimb. The time that was required to initiate movement up the ramp (initiation time) was then recorded for each forelimb. Ramp time consisted of the time required for each rat to climb the ramp. This was recorded. For the adjusting step, each rodent was held by both hindlimbs and one forelimb and moved slowly back and forth across the $0.9-\mathrm{m}$ wooden plank at a consistent speed for 30 seconds. The rodents would respond to this maneuver by rapidly taking adjusting steps with their free forelimb to keep their balance. The number of adjusting steps that were made by the rat for each specified forelimb was then recorded.

\section{Microtransplantation Procedure}

Ventral mesencephalic tissue was harvested from 14-day-old rat fetuses. The tissue was then stored at $4^{\circ} \mathrm{C}$ for 6 days in a phosphate-buffered, calcium-free hibernation medium consisting of: $30 \mathrm{mM} \mathrm{KCl}, 5$ $\mathrm{mM}$ glucose, $0.24 \mathrm{mM} \mathrm{MgCl}_{2}, 5 \mathrm{mM} \mathrm{NaH}_{2} \mathrm{PO}_{4}, 20 \mathrm{mM}$ lactic acid, $32.18 \mathrm{mM} \mathrm{KOH}$, and $164.7 \mathrm{mM}$ sorbitol, $\mathrm{pH}$ 7.4. Half of the tissue was placed in medium that was also replenished daily with human recombinant GDNF $(1 \mu \mathrm{g} / \mathrm{ml})$. The other half was stored in hibernation medium alone without GDNF. Cell suspensions were prepared in Dulbecco's modified Eagle's medium (DMEM) and dissociated in 1 $\mathrm{ml}$ of $0.1 \%$ trypsin $/ 0.05 \% \mathrm{DNase} / \mathrm{DMEM}$ at $37^{\circ} \mathrm{C}$ for 20 minutes. A final cell concentration of 
approximately 200,000 cells/ $\mu$ l was used with a viability of greater than $98 \%$, as determined by the trypan-blue dye exclusion method.

Using the micrografting technique described by Nikkhah, et al.,[21] either 400,000 or 800,000 cells were implanted into the striatum. Six deposits were made in the striatum at the following coordinates: 1) AP 1.3, L 2.1, V 5.5, and V 4.3; 2) AP 0.6, L 2.9, V 5.5, and V 4.3; and 3) AP 0.3, L 3.7, V 5.5, and V 4.3; toothbar -3.3 ; coordinates from the bregma and the ventral surface of the brain.

\section{Immunohistochemical Study}

Following completion of all behavioral testing, the animals that received the transplants were killed by an overdose of pentobarbital and perfused transcardially with $100 \mathrm{ml}$ of $0.1 \mathrm{M}$ phosphate-buffered saline (PBS) followed by $250 \mathrm{ml}$ of $4 \%$ freshly depolymerized paraformaldehyde in $0.1 \mathrm{M}$ PBS for 15 minutes. The brains were removed and postfixed overnight in 4\% paraformaldehyde in $0.1 \mathrm{M}$ PBS before being stored for 24 hours in PBS containing 30\% sucrose. Forty-micrometer-thick coronal sections were cut on a freezing microtome and collected serially in $0.1 \mathrm{M}$ PBS. Tyrosine hydroxylase (TH) immunohistochemical analysis was performed by using a primary rabbit anti-TH antiserum $(1: 2500)$ and an avidin-biotin complex (ABC) kit. Briefly, sections were prewashed in a solution of $10 \%$ methanol and $3 \%$ hydrogen peroxide for 10 minutes before being blocked in PBS containing $0.3 \%$ Triton X-100 and $5 \%$ normal swine serum for 1 hour. Sections were then incubated in a 1:2500 solution of rabbit polyclonal anti-TH antibody for 16 hours. Antibody binding was visualized by using a biotinylated swine anti-rabbit immunoglobulin antibody in a 1:500 solution that reacted with avidin-biotinylated-horseradish peroxidase (ABC kit). Peroxidase activity was developed by using a 3.3'-diaminobenzidine chromagen. Sections were washed in 0.1 M PBS, mounted on gelatin-subbed slides, dehydrated, and mounted in Permount.

\section{Cell Counts, Fiber Density, and Statistics}

Sections stained for TH were examined with the aid of the light microscope and the total number of TH-immunoreactive neurons in the grafted striatum was counted by using a $10 \mathrm{X} 10$-mm ocular lens grid. The observer was blinded to the procedence of the sections. Every fourth section was counted and an approximation of the final graft cell number was calculated by using Abercrombie's formula $[1](\mathrm{P}=$ $1 / \mathrm{f} A M[D+M])$, where $P$ is the corrected cell number, $A$ is the raw count, $D$ is the average cell diameter, $\mathrm{M}$ is the section thickness, and $\mathrm{f}$ is the frequency of selected sections. Fiber density was assessed by counting the number of TH-immunoreactive fibers crossing a 50 X 50- $\mu \mathrm{m}$ grid placed perpendicular to the graft-host interface. The number of fibers within the grid were counted at distances of 50,100,150, and $200 \mu \mathrm{m}$ from the graft-host interface. A total of 30 measurements were made for each graft as previously described.[2] All the data were analyzed using analysis of variance and t-tests with and without equal variance. Statistical analysis for between-group and within-group differences was performed using a two-way analysis of variance followed by Tukey's posthoc test where appropriate. Statistical significance was considered when the probability value was less than 0.05 .

\section{Sources of Supplies and Equipment}

The Wistar rats were purchased from Charles River Laboratories (St. Constant, Quebec, Canada). Human recombinant GDNF was obtained from Pepro Tech Inc. (Rocky Hill, NJ) and the DMEM, DNase, trypsin, 6-OHDA hydrobromide, and diaminobenzidine tetrahydrochloride tablets from Sigma Chemical Co. (Chicago, IL). Primary anti-TH was provided by Pel Freeze Biologicals (Rogers, AR) and the ABC 
kit by Vector Laboratories Canada Inc. (Burlington, Ontario, Canada). Biotinylated swine anti-rabbit immunoglobulin antibody was purchased from Dako Diagnostics Canada, Inc. (Mississanga, Ontario, Canada). The Videomex-V computerized video monitor system was purchased from Columbus Instruments (Columbus, $\mathrm{OH}$ ) and the grid used to calculate fiber density was obtained from Olympus Microscopes Carsen Group, Inc. (Markham, Ontario, Canada).

\section{RESULTS}

\section{Nigral Lesions}

Coronal sections of the ventral mesencephalon were obtained from each animal in all of the groups. The 6-OHDA lesions in the nigrostriatal dopaminergic pathway produced a virtually complete loss of TH-immunoreactive neurons and fibers in the ipsilateral substantia nigra and ventral tegmental area.

\section{Dopaminergic Grafts}

All cell suspensions were quantified and assessed for viability before implantation. All of the groups had almost identical numbers of viable cells per volume. The viability was at least $98 \%$ for all groups (Table $1)$.

\begin{tabular}{|c|c|c|c|c|}
\hline $\begin{array}{l}\text { NUMBER OF YIABLE CELL } \\
\text { IMPLANTATION OF }\end{array}$ & $\begin{array}{l}\text { TABLE } 1 \\
\text { CELL SUSPENS } \\
\text { MINERGC CELL }\end{array}$ & $\begin{array}{l}\text { N PRE } \\
\text { NTO I }\end{array}$ & $\begin{array}{l}\text { RAATION US } \\
\text { T STRMTUN }\end{array}$ & ED FOR \\
\hline Cell Suspersion & $\begin{array}{l}\text { Concertration } \\
\text { [viable } \\
\text { cells } 1 \mathrm{mln} \text { ) }\end{array}$ & $\begin{array}{l}\text { Ma- } \\
\text { bility } \\
\text { (\%) }\end{array}$ & $\begin{array}{l}\text { Yolume } \\
\text { Implant ed } \\
\text { (ml) }\end{array}$ & $\begin{array}{c}\text { No. of } \\
\text { Cells } \\
\text { Implant ed }\end{array}$ \\
\hline $\begin{array}{l}\text { control hi bernation medium } \\
\text { hibemation medium \& GDNF } \\
\text { hibemation medium \& GDNF }\end{array}$ & $\begin{array}{l}222,000 \\
242,000 \\
242,000\end{array}$ & $\begin{array}{l}98.9 \\
99.0 \\
99.0\end{array}$ & $\begin{array}{l}1.80 \\
1.65 \\
3.30\end{array}$ & $\begin{array}{l}400,000 \\
400,000 \\
800,000\end{array}$ \\
\hline
\end{tabular}

Viable grafts were observed in all groups and in all cases three graft deposits were clearly identified. Numerous TH-immunoreactive neurons were encountered in the periphery of the graft with fewer cells in the center of the graft, resembling the normal architecture of the substantia nigra with a dense pars compacta and a pars reticulata containing fewer dopaminergic neurons. (Fig. 1). A prominent network of TH-immunoreactive fibers was present within the graft and extended to variable distances into the host striatum, forming a halo of TH immunoreactivity around the grafts. 


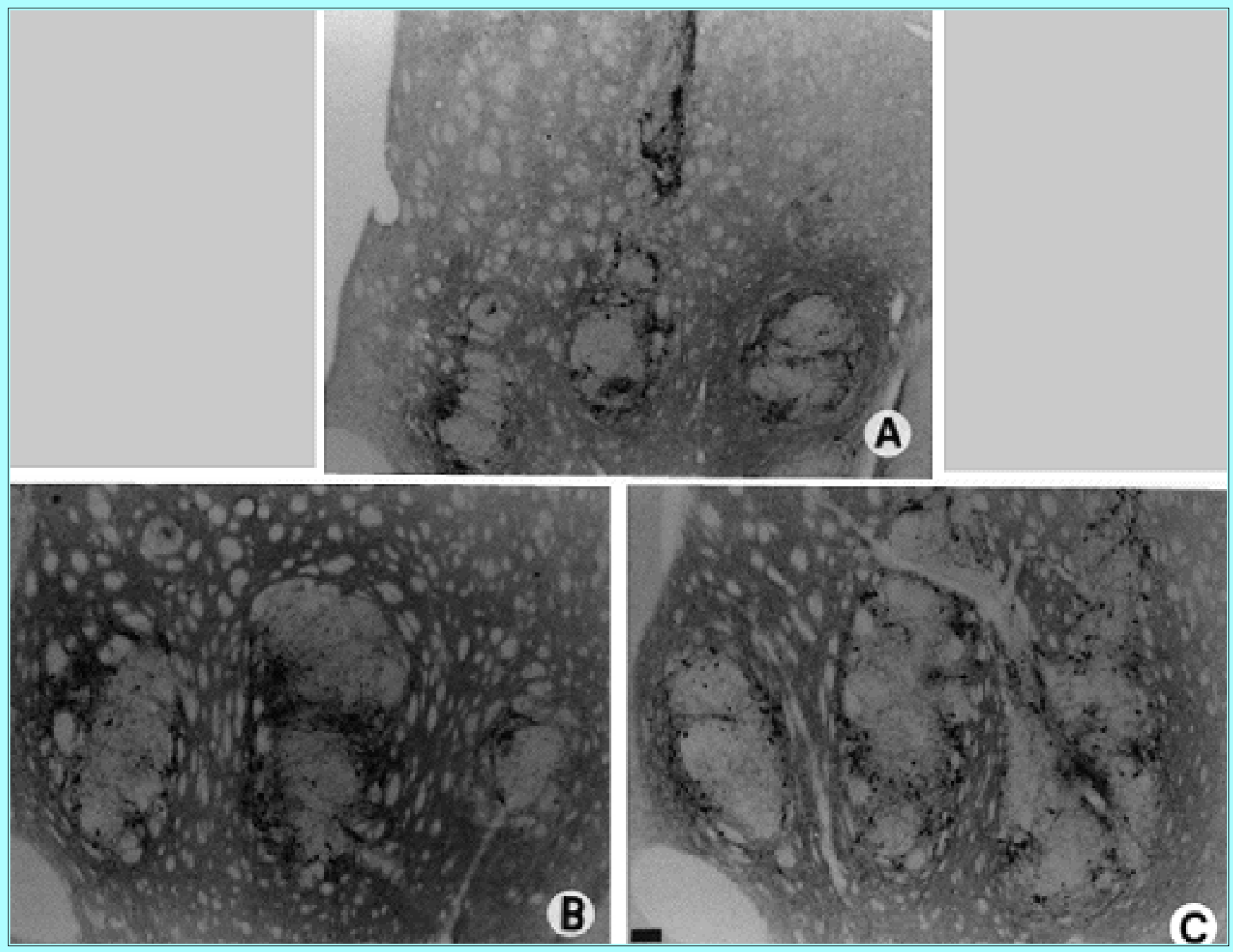

Fig. 1. Photomicrographs displaying representative TH-immunostained coronal sections of the striatum in a rat with a unilateral 6-OHDA lesion of the nigrostriatal dopaminergic pathway that was transplanted with ventral mesencephalic dopaminergic cells. A: View of a grafted striatum with 400,000 cells alone. Note three distinct grafts of TH-immunostained cells and fiber outgrowth. B: View of a grafted striatum with 400,000 cells exposed to GDNF during hibernation. Note the increase in TH-immunostaining and fiber outgrowth. C: View of a grafted striatum with 800,000 cells exposed to GDNF during hibernation. Bar $=$ $200 \mu \mathrm{m}$.

\section{Rotational Behavior}

Restoration of rotational symmetry was achieved 3 weeks posttransplantation in animals that received grafts of 400,000 cells stored in GDNF (Group 2) and in rats that received 800,000 cells that were also exposed to GDNF (Group 3) (Fig. 2). This decrease in rotations was statistically significant when compared to pretransplantation values. In contrast, rotations in animals that had grafts of 400,000 cells not exposed to GDNF (Group 1) did not change significantly at 3 weeks posttransplantation. However, this group achieved rotational symmetry at 8 weeks posttransplantation. Animals that had nigral lesions only and no grafts (Group 4) continued to show a significant clockwise (ipsilateral) rotational bias by the end of the study (10 weeks after lesioning). 


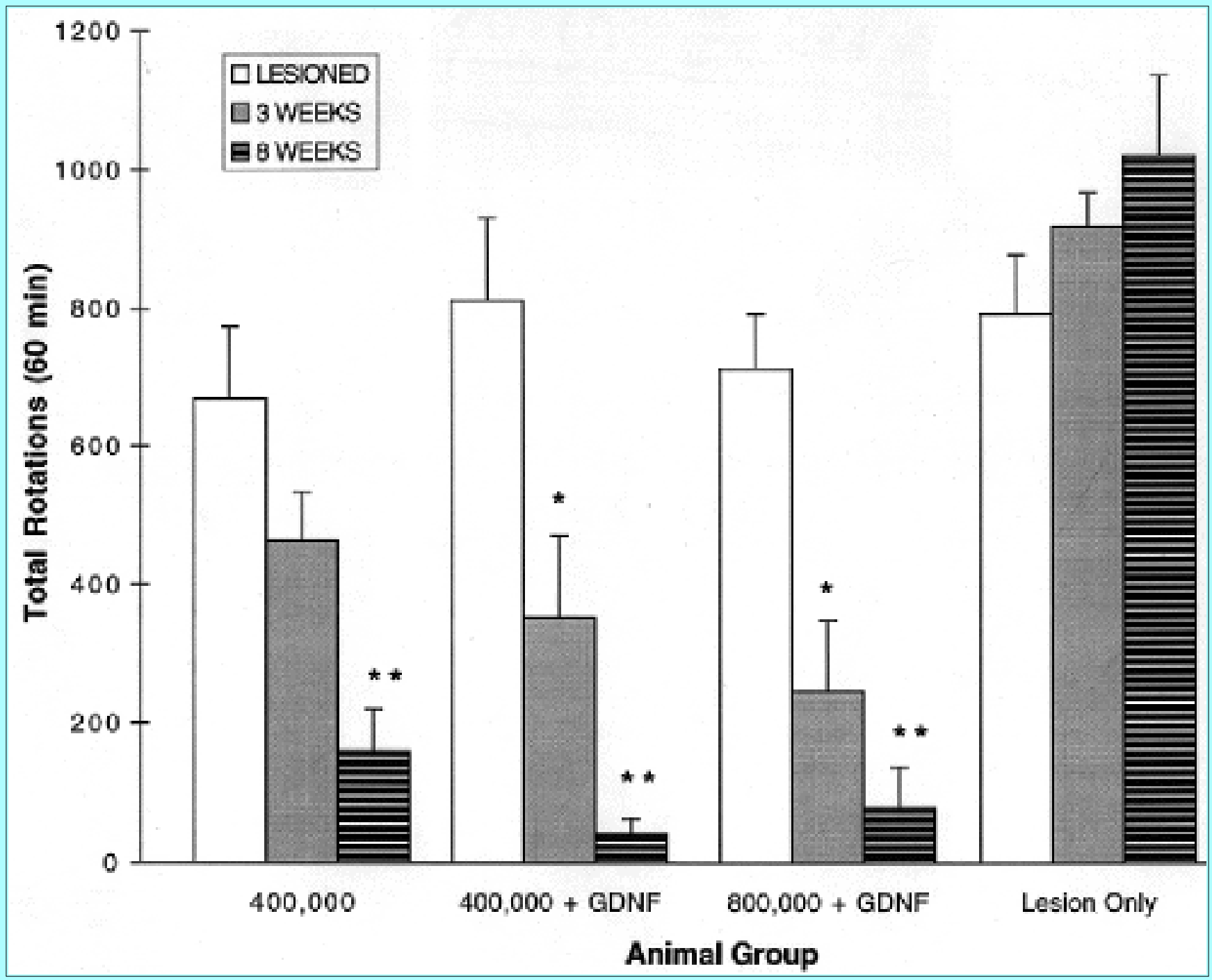

Fig. 2. Bar graph depicting rotational behavior following an amphetamine challenge (5 $\mathrm{mg} / \mathrm{kg}$ delivered intraperitoneally) after lesioning of the nigrostriatal dopaminergic pathway (open bars) and at 3 (gray bars) and 8 (striped bars) weeks posttransplantation. Animals receiving grafts of 400,000 or 800,000 cells exposed to GDNF during hibernation demonstrated a significant improvement in rotational behavior at 3 weeks posttransplantation when compared with their prelesioning values and the values of the lesion-only group $\left({ }^{*} \mathrm{p}<0.05\right)$. All animals that received transplants demonstrated significant improvement in rotation behavior at 8 weeks posttransplantation when compared with their prelesioning values and those of the lesion-only group $(* * \mathrm{p}<0.05)$.

\section{Staircase Test}

Assessment of all animals in the staircase test before lesioning revealed no bias for the use of either the right or left forelimb. On average, the rodents acquired $2.9 \pm 0.3$ and $2.5 \pm 0.6$ food pellets with their right and left forelimbs, respectively, at the 1-minute time point. These scores increased to $3.9 \pm 0.4$ and $4 \pm 0.5$ food pellets for the right and left forelimbs, respectively, at the 15-minute time point.

Following lesioning of the right nigrostriatal dopaminergic pathway, there was a consistent dropoff in left forelimb scores at the 1-minute and the 15-minute time points. The left forelimb scores at 1 minute dropped approximately $50 \%$ to $1.2 \pm 0.6$ food pellets and the 15 -minute scores dropped approximately $33 \%$ to $2.2 \pm 0.6$ food pellets. The decrease in pellet retrieval with the left forelimb after lesioning was 
statistically significant compared with the prelesion values and compared with the right forelimb. The right forelimb scores at 1 minute $(2.7 \pm 0.1$ compared with $2.9 \pm 0.3$ food pellets) and at 15 minutes (3.7 \pm 0.5 compared with $3.9 \pm 0.4$ food pellets) did not change significantly from prelesion scores.

Following transplantation, no significant improvement was observed in any of the treatment groups when tested at 8 weeks.

\section{Stepping Test}

Initiation Time. All rodents were immediately able to initiate movement with either forelimb in their course up the ramp after an initial period of training. An immediate attempt at climbing the ramp was scored as a 1-second initiation time. After lesioning the rats exhibited a very obvious impairment in their ability to initiate movement, which was more pronounced in the limb contralateral to the lesion (left forelimb) (Fig. 3). The initiation times in all transplanted groups (Groups 1, 2, and 3) for the left forelimb increased to $3.9 \pm 0.7,4.7 \pm 0.8$, and $5.5 \pm 1.2$ seconds, respectively. The initiation time for the right forelimb also increased after lesioning but was less dramatic, increasing to $2 \pm 0.3$ seconds.

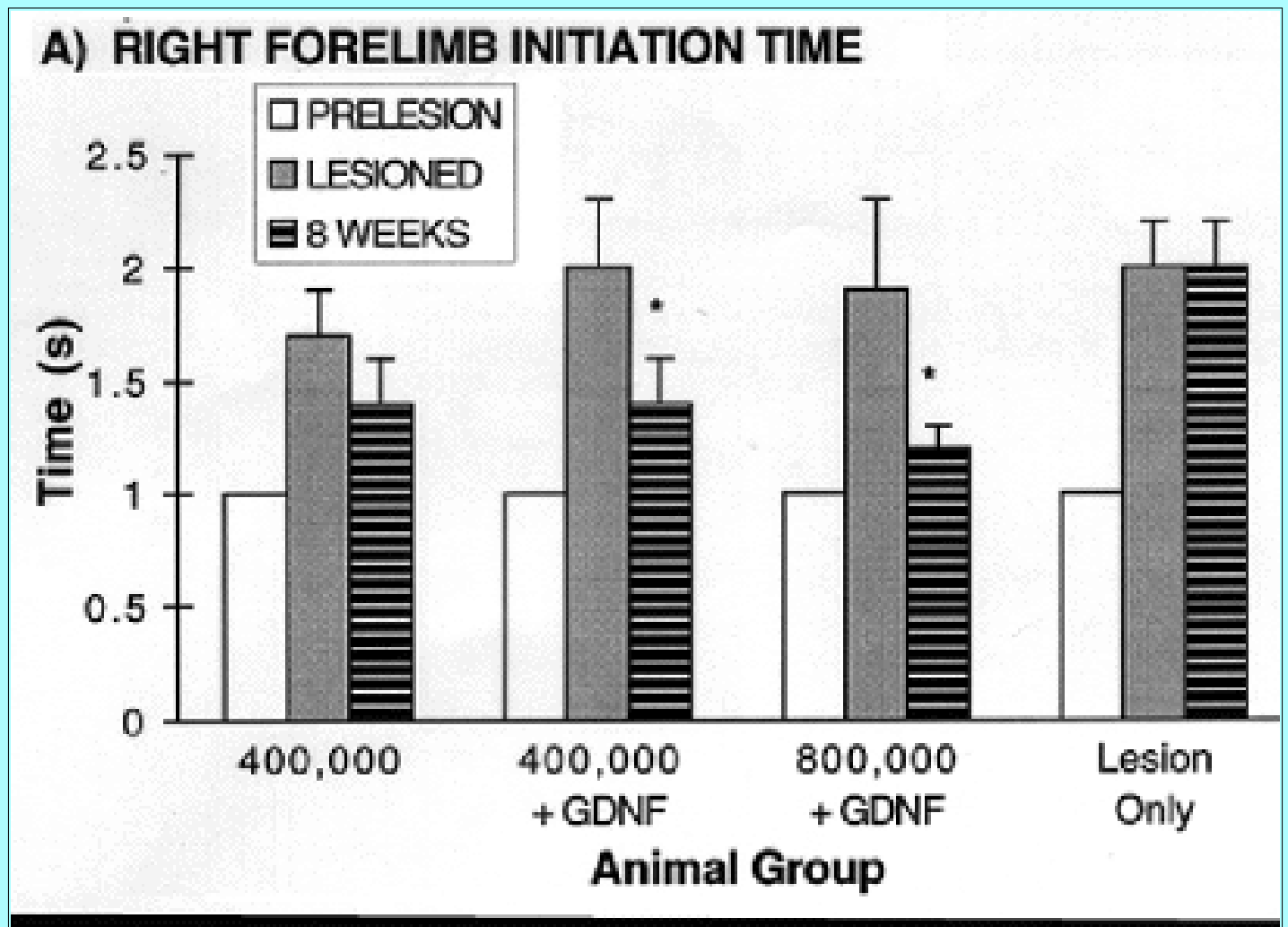

\section{B) LEFT FORELIMB INITIATION TIME}
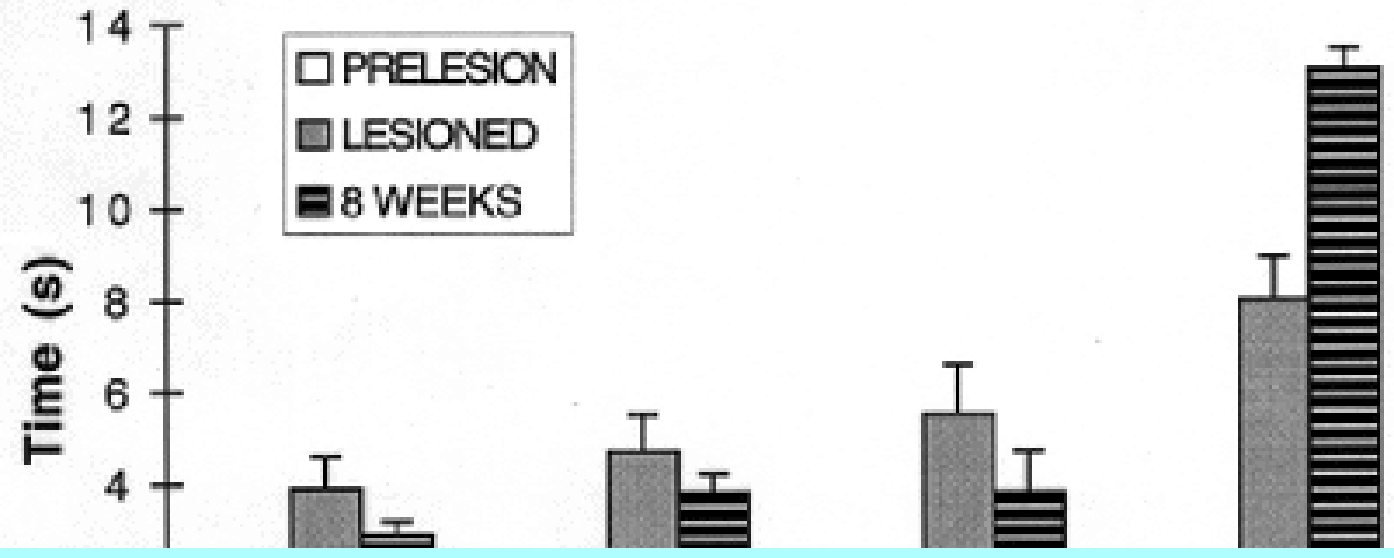


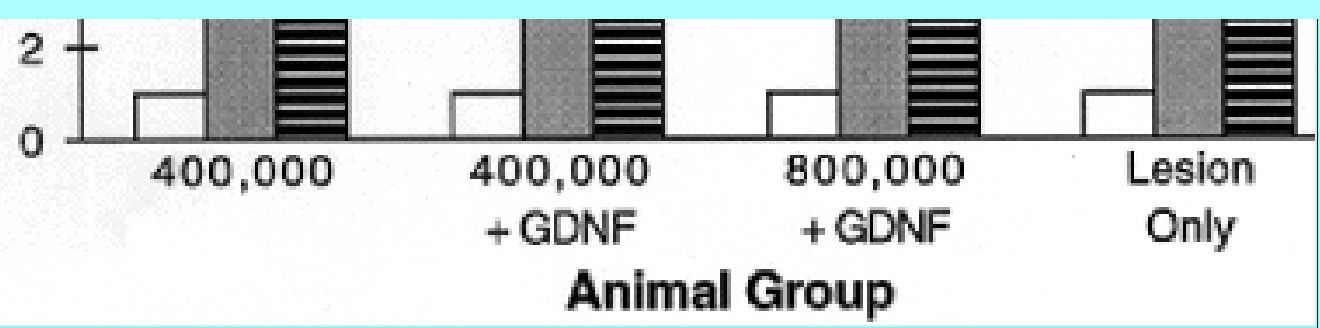

Fig. 3. Bar graphs showing forelimb initiation times (in seconds) pre- and postlesioning and at 8 weeks posttransplantation for the right (A) and left (B) forelimbs of rats with unilateral 6-OHDA lesions of the nigrostriatal dopaminergic pathway. Animals receiving grafts of 400,000 or 800,000 cells exposed to GDNF demonstrated a significant improvement in initiation time of the right forelimb compared with their postlesioning values and those of lesion-only animals $(* p<0.05)$. There was no significant improvement in initiation time of the left forelimb.

The initiation times for rodents receiving 400,000 cells not exposed to GDNF were slightly reduced after transplantation: the right forelimb initiation time was reduced from $1.7 \pm 0.2$ to $1.4 \pm 0.1$ seconds and the left forelimb initiation time was reduced from $3.9 \pm 0.7$ to $2.9 \pm 0.3$ seconds; however, these changes were not statistically significant.

Rats receiving grafts of 400,000 cells stored in GDNF showed a statistically significant improvement in right forelimb initiation time from $2 \pm 0.3$ to $1.4 \pm 0.2$ seconds. Although initiation times for the left forelimb were also reduced $(3.4 \pm 0.4$ compared with $4.7 \pm 0.8)$, this reduction did not reach statistical significance. For animals receiving 800,000 cells exposed to GDNF a significant improvement in their right forelimb initiation time from $1.9 \pm 0.4$ to $1.2 \pm 0.1$ seconds was observed after transplantation (Fig. $3 \mathrm{~A})$. Changes in the left forelimb initiation time were not significantly improved $(3.8 \pm 0.9$ compared with $5.5 \pm 1.2$ seconds) (Fig. 3B). Control animals with lesions alone exhibited increased initiation times to $2 \pm 0.2$ seconds for the right forelimb and to $13 \pm 1.2$ seconds for the left forelimb at 10 weeks postlesioning.

Ramp Time. After the training period, all rodents would quickly climb the ramp in a 2-second period. After lesioning, all ramp times substantially increased to an average of $20.3 \pm 2.5$ seconds when the right forelimb initiated the climb and to $15.3 \pm 1.9$ seconds for left forelimb initiation. No significant changes were observed after transplantation in any of the groups.

Adjusting Step. All of the rodents had achieved more than 30 adjusting steps before lesioning with no significant difference between the right and left forelimb. After lesioning, the right side adjusting step was unaffected; however, the left forelimb adjusting step was dramatically decreased. The adjusting step of the left forelimb dropped to an average of $5.3 \pm 2.5$ steps in the 30 -second test period postlesioning. During the test session, the left forelimb was flaccid and appeared to drag along the wooden plank with a lack of coordinated movement.

Eight weeks after transplantation, the rodents that received 400,000 cells without GDNF storage did not demonstrate any significant improvement in their left forelimb adjusting step $(7.9 \pm 2.4$ compared with 6 \pm 1.3 ). The left forelimb continued to drag along the test surface during the test. In contrast, animals receiving 400,000 cells exposed to GDNF demonstrated a threefold increase in their adjusting steps from $4.4 \pm 1.2$ to $17.1 \pm 2.5$ steps (Fig. 4). Although less dramatic, animals that received 800,000 cells exposed to GDNF also improved from $5.5 \pm 1.4$ steps after lesioning to $14.2 \pm 1.5$ steps following 
transplantation. In control animals with lesions alone and no grafts, no significant improvement in adjusting step was observed when tested 10 weeks postlesioning.

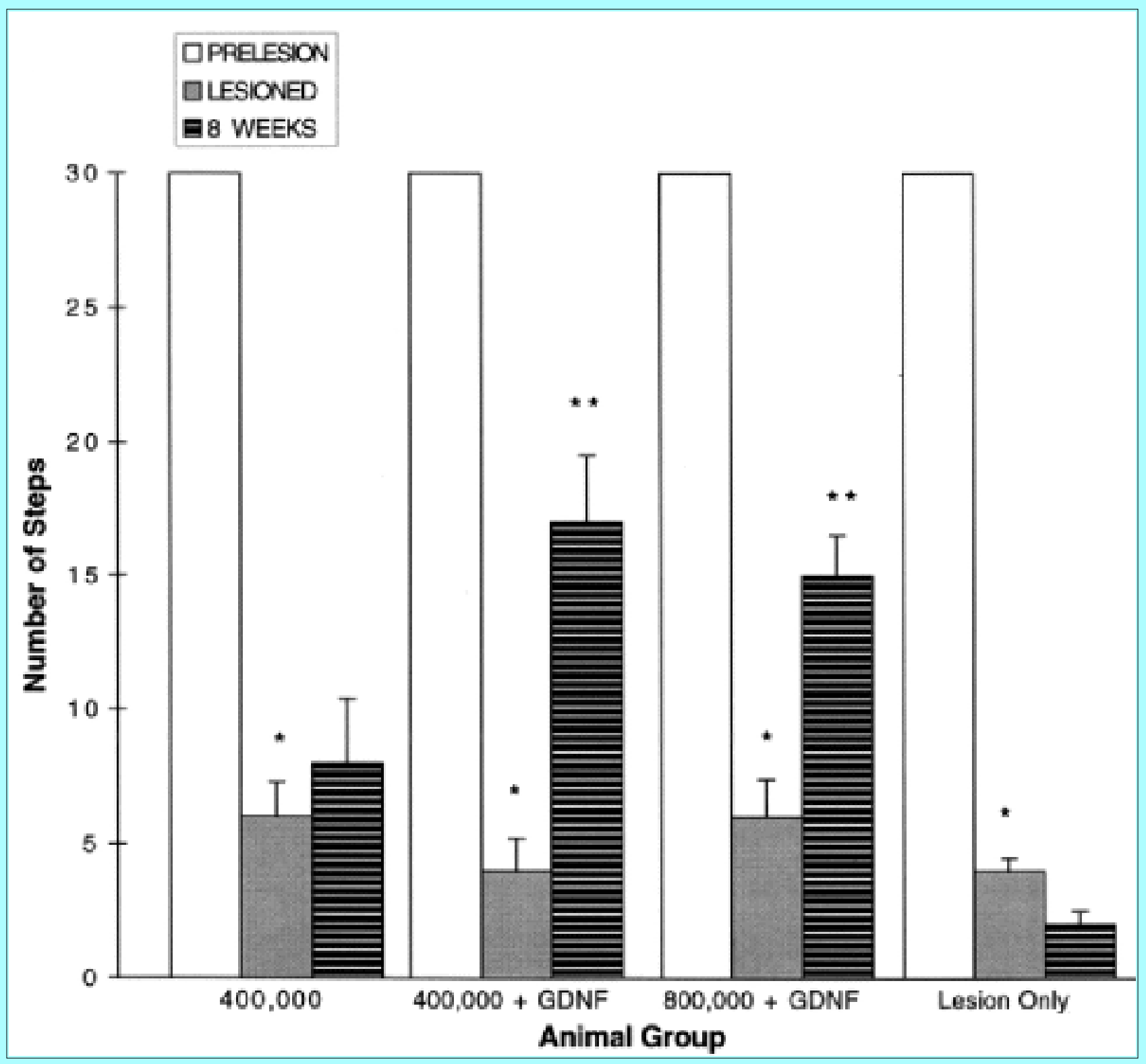

Fig. 4. Bar graph showing numbers of left forelimb adjusting steps (in the 30 -second test period) pre- and postlesioning and at 8 weeks posttransplantation for rats with unilateral 6-OHDA lesions of the nigrostriatal dopaminergic pathway. All animals demonstrated a decrease in adjusting steps following lesioning $(* p<0.05)$. Only animals that received grafts of 400,000 or 800,000 cells exposed to GDNF during hibernation demonstrated a significant improvement in left forelimb adjusting step when compared with their pretransplantation values (lesion) and those of lesion-only animals $(* * p<0.05)$.

\section{Cell Counts and Fiber Density}

Counts of TH-positive cells in grafts of animals receiving 400,000 cells not exposed to GDNF were 502 \pm 127 cells/striatum. Rats receiving the same number of cells, but exposed to GDNF, had $633 \pm 121$ cells/striatum. However, this increase in cell number did not reach statistical significance. Rats receiving 800,000 cells exposed to GDNF had the highest survival of grafted TH-immunoreactive cells at $917 \pm$ 115 cells/striatum. This increase was statistically significant when compared with the other groups. 
Similarly, the fiber outgrowth of grafts of cell suspensions exposed to GDNF during hibernation was found to be enhanced when assessed at distances of 50,100,150, and $200 \mu \mathrm{m}$ from the graft-host striatum interface when compared with the fiber outgrowth of grafts derived from cells not exposed to GDNF (Table 2).

\begin{tabular}{|c|c|c|c|c|}
\hline \multicolumn{5}{|c|}{$\begin{array}{c}\text { TABLE } 2 \\
\text { TYROSNE HYDROXYLASE IMMUNOREACTWE FIBER DENSITV IN 6-OHDA- } \\
\text { LESIONED RATS THAT RECEIYED DOPAMINERGC CELL GRAFTS* }\end{array}$} \\
\hline \multirow[b]{2}{*}{ Treatment Group } & \multicolumn{4}{|c|}{$\begin{array}{c}\text { Fiber Dersity \& } \\
\text { Distance From Graft- } \\
\text { ost Bord er }\end{array}$} \\
\hline & 501 & $100 \mathrm{~mm}$ & 150 & 20 \\
\hline \multirow{2}{*}{$\begin{array}{l}1 \text { (400,000 cells) } \\
2 \text { (400,000 cells } \\
\text { + GDNF) } \\
3 \text { (800,000 cells } \\
\text { + GDNF) }\end{array}$} & $\begin{array}{c}8.8 \pm 2.1 \\
14.3 \pm 1.9 \dagger\end{array}$ & $\begin{array}{c}6.9 \pm 1.6 \\
11.6 \pm 2.3 \dagger\end{array}$ & $6.6 \pm 1.7 \dagger$ & $\begin{array}{l}1.7 \\
6.8\end{array}$ \\
\hline & $16.8 \pm 1.6 \dagger$ & $11.9 \pm 3.7 \dagger$ & $7.8 \pm 2.9 \dagger$ & $5.9 \pm 2.3 \dagger$ \\
\hline \multicolumn{5}{|c|}{$\begin{array}{l}\text { A werage number of TH-immunoreactive fibers in the dopamine-depleted } \\
\text { striatum of } 6 \text {-OHDA-Jesioned rats grafted with dopam inergic cells treated with } \\
\text { or without GDNF for } 6 \text { days in hi bemation m edium before graft transplantation. } \\
\text { Fiber density represents the mean num ber of TH fibers } \pm \text { standard de viation } \\
\text { crossing a } 50 \times 50-m \mathrm{~m} \text { grid at distances of } 50,100,150 \text {, and } 200 \mu m \text { from the } \\
\text { graft-host striatal in terface. } \\
t p<0.05 \text { com pared with transplanted oells without GDNF treatment. }\end{array}$} \\
\hline
\end{tabular}

\section{DISCUSSION}

Numerous strategies have been used to enhance cell survival of grafted dopaminergic neurons. Grafting with glass micropipettes and small volumes of cell suspension has been reported to reduce trauma to the host tissue and increase graft survival.[20,21,23] Both antioxidants, such as lazaroids,[19] and neurotrophic factors, such as basic fibroblast growth factor,[16,33] brain-derived neurotrophic factor,[37] and GDNF,[12,32] have been demonstrated to enhance dopaminergic cell survival in in vitro and in vivo experiments; however, the use of these factors in neural transplantation has been limited. The ability of lazaroids to improve survival is believed to result from their ability to inhibit lipid peroxidation and to block free radical-induced tissue damage. Although lazaroids have been shown to have neuroprotective actions in experimental models of spinal cord injury, head trauma, and brain ischemic injury[3] and are currently being studied in subarachnoid hemorrhage,[13] their application in neural transplantation has been limited to examining their effects on graft survival following treatment at the time of transplantation[19] and not during the hibernation period required for microbiological screening in the clinical setting. On the other hand, the ability of GDNF, a polypeptide growth factor, to stimulate both survival and regrowth of dopaminergic neurons after they have been damaged has been recently demonstrated.[10] The presence of GDNF messenger RNA in dopaminergic areas including the striatum supports the concept that GDNF plays a role in the development of dopaminergic neurons.[32] Glial cell line-derived neurotrophic factor has been injected into the substantia nigra of parkinsonian rodents with mixed results in improving drug-induced rotational bias.[9,36] Recent evidence has also shown that intrastriatal GDNF in the mouse model exposed to 1-methyl-4-phenyl-1,2,3,6-tetrahydropyridine has the ability to protect nigral neurons as well as to improve rotational behavior.[34] The mechanism for this improvement likely relates to the ability of GDNF to be transported by the axon retrogradely.[35] The improvement, observed when GDNF was placed into the striatum as opposed to the nigra, supports the concept that GDNF is probably a target-derived factor normally synthesized in the striatum.[34] 
Although GDNF has been used with dopaminergic grafts,[29,30] the exposure of grafted cells to GDNF has occurred at the time of transplantation or after grafting. We have recently reported improved graft survival and fiber outgrowth when dopaminergic cells are exposed to GDNF during hibernation prior to transplantation.[2] Storage of embryonic dopaminergic tissue before implantation is important in the clinical setting because it allows time for collection of sufficient material for transplantation and completion of microbiological testing to ensure no infected material is transplanted into the recipient. In the present study, we have demonstrated that "priming" dopaminergic neurons with GDNF before transplantation not only improves graft survival but promotes functional recovery of complex sensorimotor behavior.

\section{Number of Implanted Cells and Fiber Outgrowth}

The poor cell survival rate after transplantation is one of the crucial shortcomings of neural transplantation in the clinical setting. Using current techniques, at best only 5 to $10 \%$ of grafted cells survive the implantation procedure. One of the strategies has been to increase the number of cells implanted into the host. However, this strategy has a number of disadvantages, including the problem of availability of sufficient donor fetal tissue, the necessity to increase the volume of implanted material, and the number of passes to the brain, which increases tissue trauma and the risk of intraoperative complications.

In the present study, significant improvements in rotational behavior, initiation time, and adjusting step were observed in animals receiving 400,000 or 800,000 cells exposed to GDNF during hibernation. Although the number of TH-positive cells surviving in the grafts of animals receiving 800,000 cells was higher than in grafts of animals receiving 400,000 cells (917 \pm 115 compared with $633 \pm 121$ cells/striatum), there was no significant difference in the animals' functional recovery with regard to rotation, initiation time, and adjusting step. This functional effect appears to be related to the exposure of dopaminergic cells to GDNF before transplantation, because animals that received 400,000 cells not exposed to GDNF did not exhibit significant improvement in their rotational behavior at 3 weeks or in their initiation time or adjusting step throughout the experiment, when compared to control rats that underwent lesioning but received no graft. These results also suggest that a higher number of implanted dopaminergic cells does not necessarily translate into greater functional effect even when the cells are exposed to GDNF. A possible explanation for this observation is that the functional effects of dopaminergic grafts are the result of the degree of reinnervation of the host striatum by the graft that is given by fiber outgrowth and not the number of surviving cells. Although there was enhanced fiber outgrowth from grafts derived from cells exposed to GDNF during hibernation, there was no difference in the extent of fiber outgrowth when comparing grafts in animals receiving 400,000 or 800,000 cells exposed to GDNF during hibernation. Others have reported increased fiber outgrowth in fetal dopaminergic grafts exposed to GDNF when ectopically placed in the ocular cavity[32] or the striatum,[30] and we have recently reported increased fiber outgrowth in dopaminergic grafts exposed to GDNF during storage prior to implantation in 6-OHDA-lesioned rats,[2] which is not observed in grafts acutely exposed to GDNF just prior to implantation.

\section{Sensorimotor Behavior}

Although rotational behavior has been used as the main test for functional recovery after dopaminergic transplantation in the rodent model of PD, it lacks correlation to the complex sensorimotor deficits experienced by humans with PD. Defining improvement in more complex sensorimotor deficits in 
experimental animal models may be more helpful in predicting possible clinical responses of neural transplantation strategies. The staircase test was effective in this study at demonstrating a gross reduction in the ability of the contralateral forelimb to acquire food pellets postlesioning. The inability of striatally placed grafts to overcome this deficit was demonstrated in all transplanted groups. The failure of rodents that received transplants to improve in this test may represent the inability of nigral grafts ectopically placed in the striatum to restore complex motor control. It is possible that the lack of afferent input into a nigral graft placed into the striatum is the missing factor, leading to the continued failure of transplanted animals in this test; placement of the graft into its homotopical location in the ventral mesencephalon may be necessary for an animal to display improvement on the staircase test. The importance of graft placement in this test has been demonstrated by the ability of striatal tissue in rats to improve contralateral forelimb activity after having been placed homotopically in striatum dennervated with ibotenic acid.[18]

After unilateral 6-OHDA lesioning, the initiation times in the stepping test were significantly impaired in the left forelimb compared with only a mild change in the right forelimb. These postlesioning results are consistent with the findings of other groups. [24] The lack of significant improvement after transplantation in the left forelimb's initiation ability may be caused by the failure of the intrastriatal graft to provide dopamine reinnervation to the substantia nigra and to reconstitute the nigrostriatal circuitry. This concept is supported by the observation that placement of ventral mesencephalic tissue into the nigra alone or in combination with striatal grafting did seem to improve contralateral forelimb initiation.[24] The ability to reconstitute the nigrostriatal pathway with simultaneous grafting of fetal ventral mesencephalic tissue into the substantia nigra and striatum has recently been published,[17] and we are currently investigating the ability of double dopaminergic grafts to improve initiation time and contralateral limb activity in the staircase test. Interestingly, it appears that intrastriatal grafts may be sufficient to improve a modest increase (approximately 1 second) in right forelimb initiation time. Animals receiving either 400,000 or 800,000 cells exposed to GDNF had significant improvements in their initiation times with the right forelimb when compared with animals grafted with 400,000 cells not exposed to GDNF and when compared with lesion-only control animals.

The time that was required for the rats to ascend the ramp was consistently increased postlesioning regardless of the forelimb used to initiate movement. The time was increased mainly because of the rodents' consistent behavioral bias to inspect and explore in a clockwise direction. None of the groups that underwent transplantation demonstrated any improvement in their ramp times at the 8-week time point.

The adjusting step test was the test most influenced by intrastriatal dopaminergic grafts. A dramatic change in the ability to adjust the step was observed after lesioning. The contralateral forelimb was flaccid and failed to mount adjusting steps when it was moved both in the forehand and backhand direction. Only those rodents that received grafts exposed to GDNF, which increases graft survival and fiber outgrowth,[2] demonstrated a statistically significant improvement in this test, suggesting that the adjusting step may be a striatal dopaminergic function and may not require nigral reinnervation or restoration of the nigrostriatal circuitry, which appears to be necessary in other aspects of the stepping test or the staircase test. These tests may be more complicated because they involve motivational factors and possibly more complex motor sequences that may not respond to intrastriatal dopaminergic grafts alone. $[4,22,24]$ Improvement in rotational bias and forelimb akinesia, as assessed by the adjusting step, may relate more to direct dopamine release by grafted neurons and their effect on striatal dopaminergic receptors.[24] 


\section{CONCLUSIONS}

Improving graft survival and striatal dopaminergic reinnervation are key issues to be resolved in clinical neural transplantation to maximize clinical response in parkinsonian patients. This study demonstrates that fetal dopaminergic cells exposed to GDNF before transplantation improve graft survival and complex sensorimotor behavior in their host, as assessed by the adjusting step. Furthermore, these results suggest that a higher number of implanted cells may not be necessary to increase functional recovery. This concept may have important implications in clinical fetal transplantation, which depends on the limited availability of fetal tissue. The exposure of dopaminergic cells to GDNF before transplantation may enhance the efficacy of dopaminergic grafts in improving symptoms in patients who have PD and may contribute to make neural transplantation a viable therapeutic option in the future.

\section{Acknowledgments}

The authors wish to acknowledge Mr. Damaso Sadi for his technical support and Ms. Tanya Acorn for her assistance in the preparation of this manuscript.

\section{References}

1. Abercrombie M: Estimation of nuclear population from microtome sections. Anat Rec 94:239-247, 1946

2. Apostolides C, Sanford E, Hong M, et al: Glial cell line-derived neurotrophic factor improves intrastriatal graft survival of stored dopaminergic cells. Neuroscience 83:363-372, 1998

3. Braughler JM, Hall ED, Jacobsen EJ, et al: The 21-aminosteroids: potent inhibitors of lipid peroxidation for the treatment of central nervous system trauma and ischemia. Drug Future 14:143-152, 1989

4. Dunnett SB, Whishaw IQ, Rogers DC, et al: Dopamine-rich grafts ameliorate whole body motor asymmetry and sensory neglect but not independent limb use in rats with 6-hydroxydopamine lesions.

Brain Res 415:63-78, 1987

5. Freed CR, Breeze RE, Rosenberg NL, et al: Survival of implanted fetal dopamine cells and neurologic improvement 12 to 46 months after transplantation for Parkinson's disease. N Engl J Med 327:1549-1555, 1992

6. Freed WJ, Perlow MJ, Karoum F, et al: Restoration of dopaminergic function by grafting of fetal rat substantia nigra to the caudate nucleus: long-term behavioral, biochemical, and histochemical studies. Ann Neurol 8:510-519, 1980

7. Freeman TB, Olanow CW, Hauser RA, et al: Bilateral fetal nigral transplantation into the postcommissural putamen in Parkinson's disease. Ann Neurol 38:379-388, 1995

8. Gash DM, Zhang Z, Ovadia A, et al: Functional recovery in parkinsonian monkeys treated with GDNF. Nature 380:252-255, 1996

9. Hoffer BJ, Hoffman A, Bowenkamp K, et al: Glial cell line-derived neurotrophic factor reverses toxin-induced injury to midbrain dopaminergic neurons in vivo. Neurosci Lett 182:107-111, 1994 
10. Hou JGG, Lin LFH, Mytilineou C: Glial cell line-derived neurotrophic factor exerts neurotrophic effects on dopaminergic neurons in vitro and promotes their survival and regrowth after damage by 1-methyl-4-phenylpyridinium. J Neurochem 66:74-82, 1996

11. Hudson J, Granholm AC, Gerhardt GA, et al: Glial cell line-derived neurotrophic factor augments midbrain dopaminergic circuits in vivo. Brain Res Bull 36:425-432, 1995

12. Johansson M, Friedemann M, Hoffer B, et al: Effects of glial cell line-derived neurotrophic factor on developing and mature ventral mesencephalic grafts in oculo. Exp Neurol 134:25-34, 1995

13. Kassell NF, Haley EC Jr, Apperson-Hansen C, et al: Randomized, double-blind vehicle-controlled trial of tirilazad mesylate in patients with aneurysmal subarachnoid hemorrhage: a cooperative study in Europe, Australia, and New Zealand. J Neurosurg 84:221-228, 1996

14. Kearns CM, Gash DM: GDNF protects nigral dopamine neurons against 6-hydroxydopamine in vivo. Brain Res 672:104-111, 1995

15. Lin LF, Doherty DH, Lile JD, et al: GDNF: a glial cell line-derived neurotrophic factor for midbrain dopaminergic neurons. Science 260:1130-1132, 1993

16. Mayer E, Dunnett SB, Pellitteri R, et al: Basic fibroblast growth factor promotes the survival of embryonic ventral mesencephalic dopaminergic neurons--I. Effects in vitro. Neuroscience 56:379-388, 1993

17. Mendez I, Sadi D, Hong M: Reconstruction of the nigrostriatal pathway by simultaneous intrastriatal and intranigral dopaminergic transplants. J Neurosci 16:7216-7227, 1996

18. Montoya CP, Astell S, Dunnett SB: Effects of nigral and striatal grafts on skilled forelimb use in the rat. Progr Brain Res 82:459-466, 1990

19. Nakao N, Frodl EM, Duan WM, et al: Lazaroids improve the survival of grafted rat embryonic dopamine neurons. Proc Natl Acad Sci USA 91:12408-12412, 1994

20. Nikkhah G, Bentlage C, Cunningham MG, et al: Intranigral fetal dopamine grafts induce behavioural compensation in the rat Parkinson model. J Neurosci 14:3449-3461, 1994

21. Nikkhah G, Cunningham MG, Jödicke A, et al: Improved graft survival and striatal reinnervation by microtransplantation of fetal nigral cell suspensions in the rat Parkinson model. Brain Res 633:133-143, 1994

22. Nikkhah G, Duan WM, Knappe U, et al: Restoration of complex sensorimotor behavior and skilled forelimb use by a modified nigral cell suspension transplantation approach in the rat Parkinson model. Neuroscience 56:33-43, 1993

23. Nikkhah G, Olsson M, Eberhard J, et al: A microtransplantation approach for cell suspension grafting in the rat Parkinson model: a detailed account of the methodology. Neuroscience 63:57-72, 1994

24. Olsson M, Nikkhah G, Bentlage C, et al: Forelimb akinesia in the rat parkinsonian model: differential effects of dopamine agonists and nigral transplants as assessed by a new stepping test. J Neurosci 15:3863-3875, 1995 
25. Opacka-Juffry J, Ashworth S, Hume SP, et al: GDNF protects against 6-OHDA nigrostriatal lesion: in vivo study with microdialysis and PET. Neuroreport 7:348-352, 1995

26. Perlow MJ, Freed WJ, Hoffer BJ, et al: Brain grafts reduce motor abnormalities produced by destruction of nigrostriatal dopamine system. Science 204:643-647, 1979

27. Redmond DE, Sladek JR Jr, Roth RH, et al: Fetal neuronal grafts in monkeys given methylphenyltetrahydropyridine. Lancet 1:1125-1127, 1986

28. Rioux L, Gaudin DP, Bui LK, et al: Correlation of functional recovery after a 6-hydroxydopamine lesion with survival of grafted fetal neurons and release of dopamine in the striatum of the rat.

Neuroscience 40:123-131, 1991

29. Rosenblad C, Martinez-Serrano A, Björklund A: Glial cell line-derived neurotrophic factor increases survival, growth and function of intrastriatal fetal nigral dopaminergic grafts. Neuroscience 75:979-985, 1966

30. Sinclair SR, Svendsen CN, Torres EM, et al: GDNF enhances dopaminergic cell survival and fibre outgrowth in embryonic nigral grafts. Neuroreport 7:2547-2552, 1996

31. Spencer DD, Robbins RJ, Naftolin F, et al: Unilateral transplantation of human fetal mesencephalic tissue into the caudate nucleus of patients with Parkinson's disease. N Engl J Med 327:1541-1548, 1992

32. Strömberg I, Björklund L, Johansson M, et al: Glial cell line-derived neurotrophic factor is expressed in the developing but not adult striatum and stimulates developing dopamine neurons in vivo. Exp

Neurol 124:401-412, 1993

33. Takayama H, Ray J, Raymon HK, et al: Basic fibroblast growth factor increases dopaminergic graft survival and function in a rat model of Parkinson's disease. Nat Med 1:53-58, 1995

34. Tomac A, Lindqvist E, Lin LFH, et al: Protection and repair of the nigrostriatal dopaminergic system by GDNF in vivo. Nature 373:335-339, 1995

35. Tomac A, Widenfalk J, Lin LF, et al: Retrograde axonal transport of glial cell line-derived neurotrophic factor in the adult nigrostriatal system suggests a trophic role in the adult. Proc Natl Acad Sci USA 92:8274-8278, 1995

36. Winkler C, Sauer H, Lee CS, et al: Short-term GDNF treatment provides long-term rescue of lesioned nigral dopaminergic neurons in a rat model of Parkinson's disease. J Neurosci 16:7206-7215, 1996

37. Yurek DM, Lu W, Hipkens S, et al: BDNF enhances the functional reinnervation of the striatum by grafted fetal dopamine neurons. Exp Neurol 137:105-118, 1996

Manuscript received August 6, 1997.

Accepted in final form January 7, 1998.

This article was previously published in the Journal of Neurosurgery, Volume 88, pages 1088-1095. Address reprint requests to: Ivar Mendez, M.D., Ph.D., Neural Transplantation Laboratory, Room 12H1, Sir Charles Tupper Medical Building, Dalhousie University, Halifax, Nova Scotia, B3H 4H7 Canada. 